\title{
Botulinum toxin type A for painful diabetic neuropathy: an open-label study
}

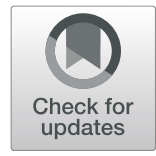

Shahinaz Helmy*, Tamer Emara and Amr Abdel Menem

\begin{abstract}
Background: A large proportion of painful diabetic neuropathy cases either do not respond or are intolerant to the currently available oral and physical therapies. There is encouraging evidence from a small number of studies that those patients can improve using botulinum toxin injection. The aim of the study was to evaluate the effect of intradermal injection of botulinum toxin type A on painful diabetic neuropathy. Eight adult patients with diabetic peripheral neuropathy (DPN), confirmed by nerve conduction studies, were refractory to a minimum of two neuropathic pain treatments for 6 months or more were recruited. All cases received intradermal injection of 48 units of botulinum toxins - type A in each foot in $6 \times 4$ distribution. Follow-up was done after 8 weeks using the Neuropathic Pain Scale (NPS), Pittsburgh Sleep Quality Index (PSQI), and Overall Disability Sum Scale (ODSS).

Results: After 8 weeks, there was a significant change in NPS from 55.8 (7.8) to 38.5 (8.1) ( $p$ value 0.007), also there was significant improvement in ODDS from $4(\mathrm{IQR}, 2.25-4.75)$ to 2.5 (IQR, 0.5-3) ( $p$ value 0.01$)$. However, there was no significant change in PSQI.

Conclusion: Botulinum toxin type A injection is a promising treatment option in Egyptian DPN adults in this study.

Keywords: Botulinum toxin type A, Diabetic peripheral neuropathy
\end{abstract}

\section{Background}

Diabetic peripheral neuropathy (DPN) is one of the most common complications of diabetes mellitus (DM). It involves signs and symptoms of peripheral neuropathy in diabetic patients after exclusion of other possible causes [1]; it nearly affects $50 \%$ of diabetic patients during their life time $[1,2]$. The prevalence of DPN varies in the literature (6-51\%) depending on type of diabetes, duration, and the population studied [3], another recent report found the overall prevalence to be around 40.3\% [4].

Painful diabetic neuropathy affects about 16\% of diabetic patients [5], and in another report the neuropathic pain symptoms were found among $10-30 \%$ of affected patients [3]. This was frequently unreported or untreated and usually affects patient's quality of life and sleep. The patients usually complain of lancinating pains, burning,

\footnotetext{
*Correspondence: Drshahinaz82@gmail.com

Neurology Department, Faculty of Medicine, Ain Shams University, Cairo, Egypt
}

or deep-seated ache that affects mainly the lower extremities [6].

Many symptomatic treatments are used for the relief of pain, like antidepressants, anticonvulsants, and opioids. However, sometimes these medications are not effective, or the patients could not tolerate higher doses or side effects. This necessitated the trial of another option that could have prolonged effect and less side effects [7].

Previous trials on botulinum toxin in the treatment of patients with diabetic neuropathy had shown promising results [6-8]. Botulinum toxin (BoNT) has been used for many decades in the treatment of spasticity and dystonia. Aside from the muscle relaxant effect, it also was used to decrease pain in cases such as trigeminal neuralgia, carpal tunnel syndrome (CTS), post herpetic neuralgia, and other types of neuropathic pains [9]. Its analgesic effect is thought to be due to inhibition of neurotransmitters release and inflammatory mediators from sensory nerves, such as serotonin, glutamate, glycine, CGRP, substance p, and many others [9].
SpringerOpen

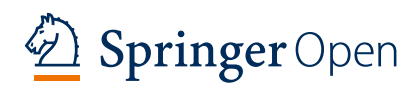

(c) The Author(s). 2021 Open Access This article is licensed under a Creative Commons Attribution 4.0 International License, which permits use, sharing, adaptation, distribution and reproduction in any medium or format, as long as you give appropriate credit to the original author(s) and the source, provide a link to the Creative Commons licence, and indicate if changes were made. The images or other third party material in this article are included in the article's Creative Commons licence, unless indicated otherwise in a credit line to the material. If material is not included in the article's Creative Commons licence and your intended use is not permitted by statutory regulation or exceeds the permitted use, you will need to obtain permission directly from the copyright holder. To view a copy of this licence, visit http://creativecommons.org/licenses/by/4.0/. 
Our study aimed to evaluate the effect of intradermal injection of botulinum toxin type $\mathrm{A}$ in pain associated with diabetic peripheral neuropathy in an Egyptian sample and its effect on disability and functional outcome.

\section{Methods}

An open-label study, that included eight adult patients with a history of $\mathrm{DM}$, and diabetic neuropathy that was diagnosed clinically and by nerve conduction study which showed decreased amplitude of compound muscle action potential (CMAP), or sensory nerve action potential (SNAP) of peroneal, tibial, or sural nerve below normal limit suggestive of axonal distal polyneuropathy of lower limbs. Nerve conduction study was done using $\mathrm{Ni}$ colet Viking Quest machine, Natus, USA.

The pain was refractory to a minimum of two neuropathic pain treatments (anti-epileptics: carbamazepine, gabapentin, and tricyclic antidepressants) for 6 months or more. Patients with other causes of pain in lower limbs, other causes of peripheral neuropathy, lumbosacral radiculopathy, or had contraindications for botulinum toxin were excluded from the study. A written informed consent was obtained from all patients with the right to withdraw from the study at any time.

All cases received intradermal injection of 48 units of botulinum toxin type $\mathrm{A}$ in each foot in $6 \times 4$ distribution. Assessment was done before injection by using the Neuropathic Pain Scale (NPS) and Pittsburgh Sleep Quality Index (PSQI) and Overall Disability Sum Score (ODSS) leg section. Follow-up of these scales was done after 8 weeks from injection.

Assessment of any side effects was done during firstweek follow-up, by asking the patients and looking for any local reaction.

NPS presents 10 domains of pain, including 2 items that assess global pain intensity, and pain unpleasantness, 8 items that assess qualities or locations of neuropathic pains; subjects were asked to rate each quality of pain on scale from $0-10$, where 0 no pain, 10 the most severe pain.

ODSS is a scale used for assessment of disability associated with neuropathy; we used the leg section to evaluate the degree of disability. In the lower limb section, it is scored from $0-7$, where 0 indicates no disability while 7 the most disability.

\section{Statistical analysis}

The data was analyzed with Statistical Package for Social Science (SPSS) version 20 (by IBM: Armonk; New York, USA). Descriptive statistics were displayed as mean and standard deviation (SD) for continuous data and case count and percentage for categorical data. Within the group, comparisons were done using the paired $T$ test and Wilcoxon signed-rank test for parametric and non- parametric data respectively. The level of statistical significance ( $p$ value) was set at 0.05 .

\section{Results}

The study involved 8 patients; the demographic data was shown in Table 1. Table 2 shows NPS, PSQI, and ODSS baseline and follow-up after 8 weeks.

Our study showed a significant change in NPS after 8 weeks follow-up from botulinum toxin injection, from 55.8 (7.8) to 38.5 (8.1) ( $p$ value 0.007). Also, there was significant improvement in ODSS from 4 (IQR, 2.254.75 ) to 2.5 (IQR, 0.5-3) ( $p$ value 0.01 ). PSQI shows improvement but was not statistically significant $(p=0.1)$ (Table 2).

There were no reported side effects of BoNT injection among the studied patients.

\section{Discussion}

Diabetic neuropathy is one of the major complications of diabetes, many drugs are used to decrease neuropathic pains in patients with diabetic neuropathy; however, sometimes it is not effective or patients cannot tolerate the side effects. Botulinum toxin was used in the treatment of several pain conditions, and many trials in diabetic neuropathy show promising results.

Our study is an open-label study aimed to evaluate the effect of intradermal injection of botulinum toxin type A in patients with painful diabetic neuropathy. We found that there was significant improvement in NPS scale after 8 weeks follow-up. This result was in favor with previous studies. Ranoux and colleagues studied the effect of botulinum toxin injection; they found significant effect on pain intensity of diabetic peripheral neuropathy from 2-12 weeks after injection, they concluded that botulinum toxin had an analgesic effect independent of the effect on muscle tone [10].

Also, Yuan and colleagues found significant reduction in diabetic painful neuropathy using visual analog score (VAS) during a 12 -week period, and $44 \%$ of their patients show reduction in pain during 3 months after injection [11]. In 2014, Ghasemi and colleagues also found significant improvement in NPS, VAS in injected group versus placebo [7]. A more recent study in 2020 included 42 patients with DPN; they found significant reduction in Visual Analog Scale (VAS), 1, 4, and 12

Table 1 Demographic data

\begin{tabular}{llll}
\hline Sex & Male, $\mathbf{n}(\%)$ & $\mathbf{4}$ & $\mathbf{5 0 \%}$ \\
& Female, $\mathbf{n ~ ( \% ) ~}$ & $\mathbf{4}$ & $\mathbf{5 0 \%}$ \\
\hline Age (mean, SD) & 57.1 & 8.9 \\
Duration of DM (mean, SD) & 18.4 & 5.5 \\
HbA1c (mean, SD) & 10.2 & 1.4
\end{tabular}

SD standard deviation, $n$ number, DM diabetes mellitus 
Table 2 NPS, ODSS, PSQI before and after botox injection

\begin{tabular}{llll}
\hline Scale & Before botox & After botox & $\boldsymbol{P}$ value \\
\hline NPS (mean, SD) & $55.8(7.8)$ & $38.5(8.1)$ & $0.007^{*}$ \\
ODSS (median, IQR) & $4(\mathrm{IQR}, 2.25-4.75)$ & $2.5(0.5-3)$ & $0.01^{*}$ \\
PSQI (mean, SD) & $14.6(4.1)$ & $11.8(3.1)$ & 0.1 \\
\hline
\end{tabular}

IQR interquartile range, SD standard deviation, NPS Neuropathic Pain Scale, ODSS Overall Disability Sum Score, PSQI Pittsburgh Sleep Quality Index *Significant

weeks after injection in patients injected with botulinum toxin in comparison with placebo group [6].

We also found a significant improvement in ODSS ( $p$ value 0.01 ), and to our knowledge this assessment was not done on the previous studies. This finding indicates that improvement of pain in DPN was reflected also on the functional disability and quality of life. The results regarding PSQI were not significant; this was also the case in Egila and colleagues, they did not find significant changes in PSQI, while Yuan and colleagues found significant improvement. This could be due to the sample size $[6,11]$.

Our study had some limitations; it was an open-label study, so the small sample size and no control group were the main limiting points. So, further case control with large sample size is needed for better evaluation.

\section{Conclusion}

Our study found a significant improvement in pain in DPN, after using intradermal injection of botulinum toxin. This gives a new hope for the management of painful diabetic neuropathy with minimal side effects.

\section{Abbreviations}

BoNT: Botulinum toxin; CGRP: Calcitonin gene-related peptide; CMAP: Compound muscle action potential; DM: Diabetes mellitus; DPN: Diabetic peripheral neuropathy; NPS: Neuropathic Pain Scale; ODDS: Overall Disability Sum Score; PSQI: Pittsburgh Sleep Quality Index; SD: Standard deviation; SNAP: Sensory nerve action potential; SPSS: Statistical Package for Social Science; VAS: Visual Analog Scale

\section{Acknowledgements}

Not applicable

\section{Authors' contributions}

$\mathrm{AA}, \mathrm{TE}$, and $\mathrm{SH}$ conceived of the study and participated in its design and coordination and helped to draft the manuscript. TE and SH participated in the design of the study and performed the statistical analysis. AA injected the botulinum toxin for the patients. All authors have agreed to conditions noted on the Authorship Agreement Form. The authors read and approved the final manuscript.

\section{Funding}

Self-financing.

\section{Declarations}

\section{Ethics approval and consent to participate}

The study protocol was approved by Ain Shams University, Faculty of Medicine Research Ethical Committee of Neurology Department (on 19 January 2020). Written informed consent was obtained from the patients participating in the study after informing them about the study rationale and their right to withdraw from the study at any time without any consequences. Committee's reference number-not applicable.

\section{Consent for publication}

Not applicable

\section{Competing interests}

All authors declare that they do not have any competing interests.

Received: 12 February 2021 Accepted: 4 May 2021

Published online: 17 May 2021

\section{References}

1. Zeng L, Alongkronrusmee D, Van RM. An integrated perspective on diabetic, alcoholic, and drug-induced neuropathy, etiology, and treatment in the US. J Pain Res. 2017;10:219-28. https://doi.org/10.2147/JPR.S125987.

2. Edwards JL, Vincent AM, Cheng HT, Feldman EL. Diabetic neuropathy: mechanisms to management. Pharmacol Ther. 2008;120(1):1-34. https://doi. org/10.1016/j.pharmthera.2008.05.005

3. Hicks C, Selvin E. Epidemiology of peripheral neuropathy and lower extremity disease in diabetes. Curr Diab Rep. 2019;19(10):86-99. https://doi. org/10.1007/s11892-019-1212-8.

4. Pfannkuche A, Alhajjar A, Ming A, Walter I, Piehler C, Mertens P. Prevalence and risk factors of diabetic peripheral neuropathy in a diabetics cohort: register initiative "diabetes and nerves". Endocr Metab Sci. 2020;1(1-2): 100053. https://doi.org/10.1016/j.endmts.2020.100053.

5. Djibril AM, Mossi EK, Djagadou AK, Balaka A, Tchamdja T, Moukaila R. Epidemiological, diagnostic, therapeutic and evolutionary features of diabetic foot: a study conducted at the medico-surgical clinic. University Hospital Sylvanus Olympio in Lome. Pan Afr Med J. 2018;30:4.

6. Egila H, Gomaa M, Abdel-Naby A, Shehab M. Botulinum toxin type A in severe diabetic neuropathy. Benha Med J. 2020;37(3):723-30.

7. Ghasemi M, Ansari M, Basiri K, Shaigannejad V. The effects of intradermal botulinum toxin type $A$ injections on pain symptoms of patients with diabetic neuropathy. J Res Med Sci. 2014;19(2):106-11.

8. Jabbari B, Safarpour Y. Botulinum toxin treatment for painful diabetic neuropathy: a review. IJAPR. 2019;1(1):1-4.

9. Park J, Park HJ. Botulinum toxin for the treatment of neuropathic pain. Toxins. 2017:9(9):1-15.

10. Ranoux D, Attal N, Morain F, Bouhassira D. Botulinum toxin type A induces direct analgesic effects in chronic neuropathic pain. Ann Neurol. 2008;64(3): 274-83. https://doi.org/10.1002/ana.21427.

11. Yuan RY, Sheu JJ, Yu JM, Chen WT, Tseng IJ, Chang HH, et al. Botulinum toxin for diabetic neuropathic pain: a randomized double -blind crossover trial. Neurology. 2009;72(17):1473-8. https://doi.org/10.1212/01.wnl.000034 5968.05959.cf

\section{Publisher's Note}

Springer Nature remains neutral with regard to jurisdictional claims in published maps and institutional affiliations. 